\title{
Rational dispensing and use of artemether-lumefantrine during pregnancy in Dar es Salaam, Tanzania
}

\author{
APPOLINARY R. KAMUHABWA* and FATEMA MNYUSIWALLA \\ Unit of Pharmacology and Therapeutics, School of Pharmacy, Muhimbili University of Health and Allied \\ Sciences, P.O. Box 65013, Dar es Salaam Tanzania
}

\begin{abstract}
Artemether-Lumefantrine (ALu) is widely used for uncomplicated malaria during the second and third trimester of pregnancy. Because of the suspected teratogenic effects of artemether during the first trimester, quinine is used in early pregnancy unless the risks outweigh the benefits. The aim of this study was to assess dispensing practice of ALu in private pharmacies and knowledge of pregnant women regarding the use of ALu. This was a prospective-descriptive study involving visits to 200 private retail pharmacies (using a mystery shopper) and interviewing pregnant women at the municipal public hospitals in Dar es Salaam, Tanzania. Among the drug dispensers, 60 (30\%) were pharmacists, 71(35.5\%) nurse assistants, $34(17 \%)$ pharmaceutical technicians and $35(17.5 \%)$ sales persons with no formal education on drug dispensing. Among the dispensers, $14.5 \%$ had high knowledge, $38.0 \%$ had medium knowledge and $47.5 \%$ had low knowledge on the use of ALu during pregnancy. About thirty three percent of the drug dispensers were willing to dispense ALu during the first trimester of pregnancy. Sixty two percent of the drug dispensers indicated that ALu is the drug of choice for uncomplicated malaria after the first trimester of pregnancy. However, 36\% indicated that ALu could not be used during pregnancy. A total of 200 pregnant women were interviewed. Among them, 16.5\% were aware that ALu should not be taken during the first trimester of pregnancy. Only 17\% of pregnant women were given information on the importance of taking food when using ALu, but none of them was given information on the importance of fatty meals when using ALu. In conclusion, the results show that most drug dispensers have inadequate knowledge about good dispensing practice of ALu in pregnancy. There is therefore a need for continuing training of drug dispensers regarding antimalarial drugs use in pregnancy.
\end{abstract}

Key words: Artemether, lumefantrine, dispensers, pregnant women, malaria, Tanzania

\section{Introduction}

In response to widespread resistance of Plasmodium falciparum to commonly used antimalarial monotherapies, particularly chloroquine and sulfadoxine-pyrimethamine (SP), many countries have recently adopted artemisinin-based combination therapy (ACT) as the first-line regimen for the treatment of uncomplicated malaria (Worrall et al., 2007). Tanzania changed its malaria treatment policy from chloroquine to SP as the first line drug in August 2001. The change was also accompanied with the re-introduction of amodiaquine as second line drug and quinine remained as the drug of choice for the treatment of severe malaria. The shift was necessary following research results, which indicated very high malaria parasite resistance to chloroquine (Draper et al., 1985, 1988 ; Kilimali \& Mkufya, 1985; Kitua, 1999). From 1996, high SP resistance rates were reported from a number of studies in Tanzania (Rønn et al., 1996; Mugittu et al.,

$*$

Correspondence: Dr. A.R. Kamuhabwa; E-mail: akamuhabwa@muhas.ac.tz; apporug@yahoo.com 
2004). As a result of $P$. falciparum resistance to SP, artemether-lumefantrine (ALu) replaced SP as the first-line regimen for malaria in 2006.

WHO recommends that uncomplicated malaria during the second and third trimester of pregnancy be treated with short course (3 day) fixed-dose of ACT. However, treatment with ACT in the first trimester is not recommended because of concerns raised by animal experiments which suggested that artemisinins might be teratogenic and cause foetal resorption if given during early gestation (WHO, 2008). Further studies have confirmed the embryotoxic effects of artemisinin and its derivatives in animals, including primates, with risk being confined to a defined period of gestation (McGready et al., 2006). The teratogenic effect is thought to involve red blood cells production, which implies the human sensitive period would be within the first trimester of pregnancy (Laffan et al., 2006). The recommended treatment of malaria in the first trimester is oral quinine.

Artemether and lumefantrine differ markedly in terms of rate of absorption and elimination. Artemether is absorbed rapidly, reaching a peak concentration at approximately two to three hours after dosing (White et al., 1999). In contrast, lumefantrine is absorbed and cleared more slowly, acting to eliminate the residual parasites (van Vugt et al., 2000). The absorption of lumefantrine is influenced by lipids and food intake. This is because lumefantrine is a lipophilic compound and fatty food enhances solubilisation and penetration of the drug through the membranes (Kokwaro et al., 2007). Therefore drug dispensers should be aware of this and counsel patients to take the medication with fatty meals.

In Tanzania, private retail community pharmacies and drug stores play a central role in the provision of malaria treatment, partly complementing public health facility services where these are unable to deliver (Hetzel et al., 2008). In Dar es Salaam, the commercial capital of Tanzania, almost all the retail community pharmacies dispense ALu to patients including pregnant women. Ideally, pharmacists should be available for dispensing of medications especially "prescription only medicines" in the community pharmacies. However, this has not been the case in most developing countries, including Tanzania (Minzi et al., 2008). It can therefore be concluded that untrained staff called 'dispensers' run and supervise these premises. While the use of these premises improves accessibility to medicines to many people, it compromises heavily on quality, efficacy and safety of drugs, the fundamentals of pharmaceutical care.

Dispensing of $\mathrm{ALu}$, especially in pregnant women without consideration for contraindication during the first trimester may result in unwanted effects to the unborn babies. In addition, irrational dispensing of ALu without taking into account the drug-food interactions might lead to emergence of resistance of malaria parasites towards ALu in the population. This study was therefore carried to assess rational dispensing of ALu during pregnancy with respect to advice given to patients about meals, contraindication during the first trimester and dosage of ALu. 


\section{Materials and Methods}

\section{Study site}

This study was carried out between December 2009 and May 2010 in Dar es Salaam, Tanzania. Dar es Salaam is divided into three municipalities (Kinondoni, Ilala and Temeke). It is the largest business city in Tanzania with a population of about 4 million people. The City has the highest number of pharmaceutical outlets compared to other regions in Tanzania.

\section{Study design and data collection}

An inventory containing a list of registered pharmacies in Dar es Salaam was obtained from Tanzania Food and Drugs Authority (TFDA). In September 2009, a total of 233 retail community pharmacies were registered in Dar es Salaam; of these, 110 were in Kinondoni, 96 in Ilala and 27 in Temeke municipalities. A total of 200 pharmacies were randomly selected for inclusion in the study. It was however, observed that pharmacies were more concentrated in some areas than others. Therefore, after random selection the list of selected pharmacies was reviewed and pharmacies selected again in accordance with their geographical representation within the municipality. In each municipality, the selected pharmacies were stratified according to their location (city centre or peri-urban). Only retail pharmacies that were dispensing ALu (Coartem $\left.{ }^{\circledR}\right)$ were involved in the study.

A simulated client (mystery shopper) method (Weiss et al., 2010) was used to collect information. In the present study, a fourth year pharmacy female student visited community pharmacies posing as pregnant woman with symptoms of malaria and enquired to be advised on the use of ALu. The simulated client used a designed form containing questions that were designed to gather information with regard to dispenser's knowledge about contraindication of ALu during pregnancy, importance of meals and fatty meals when dispensing ALu to patients, patient counselling on the timing of the doses of ALu and possible ALu-induced side effects. Immediately after leaving the pharmacy, the simulated client filled-in responses from the dispenser in the checklist form.

The filled-in information in the checklist was used to assess dispensing knowledge of the drug dispensers. A knowledge scale was prepared; one point was awarded for each correct answer and a zero point for a wrong answer. The drug dispenser's knowledge was then graded as low (0-1), medium (2-3) and high (4-5) on the basis of the questions in the check list. The questions were mainly based on the knowledge on the use of ALu during pregnancy, dosage regimen, importance of meals and fatty meals when taking $\mathrm{ALu}$, and alternative antimalarial drug which is recommended during the first trimester of pregnancy.

The main target was to assess the knowledge of pharmaceutical personnel (pharmacists, pharmaceutical technicians and pharmaceutical assistants), specifically those who are directly involved in dispensing medicines. If no pharmaceutical personnel were available in the pharmacy, other categories of sales persons involved in the dispensing of medicines were also involved in the study.

Amana, Temeke and Mwananyamala hospitals, which are located in Ilala, Temeke and Kinondoni Municipalities, respectively were visited to interview pregnant women attending antenatal clinics and receiving malaria treatment. Selection of pregnant women for the study 
was done using a convenient method i.e. any pregnant woman attending antenatal clinic for malaria treatment and willing to be interviewed was included in the study. This method was used because of the limited number of pregnant women with malaria attending the antenatal clinics in the selected hospitals.

Questionnaires were divided in two parts; the social demographic information of the respondents and questions designed to assess awareness and knowledge of pregnant women on the use of ALu during pregnancy. Social demographic information included: age, weeks of pregnancy, level of education, parity and occupation. The correctness on the dosages of ALu was assessed according to the dose schedules for adults (MoHSW, 2006).

\section{Data analysis}

Data collected using checklists and questionnaires were coded and entered in the computer for analysis using Epi Info computer program version 3.4. Data analysis was done using descriptive statistics including frequencies and cross tabulations. Chi square was used to calculate $p$-values which were then utilized for measuring the significance of differences for the study variables. Results were considered statistically significant if $p$-value of less than 0.05 was obtained.

\section{Ethical considerations}

Because of the nature of this study drug dispensers were not informed in advance and asked for consent to participate in the study. All the information obtained was kept strictly confidential, and none of the dispenser's responses were reported to the pharmacy owners. No names of the dispensers were recorded in the questionnaire and data were entered into the computer using only study code numbers. In the case of pregnant women, informed consent was obtained from the study participants. The study was granted ethical clearance from Muhimbili University of Health and Allied Sciences Research and Publications Committee. Permission to conduct the study at the selected retail community pharmacies and public health facilities was obtained from TFDA and Municipal Medical Officer in-charge, respectively.

\section{Results}

\section{Drug dispensing personnel}

Among the 200 drug dispensers, 60 (30\%) were pharmacists, 34 (17\%) pharmaceutical technicians, 71 (35.5\%) nurse assistants and 35 (17.5\%) sales persons with no formal education on drug dispensing. One hundred and thirty nine $(69.5 \%)$ drug dispensers enquired about the age of pregnancy before dispensing ALu. The rest $(30.5 \%)$ were willing to dispense ALu regardless of the age of pregnancy. After a simulate client mentioned that her pregnancy was two months old, 65 (32.8\%) dispensers were willing to dispense ALu. The rest 135 (67.5\%) were reluctant to dispense ALu mentioning that it can cause harmful effects to the foetus including foetal growth retardation $(24.8 \%)$, harmful effects to the foetus $(70.7 \%)$ and miscarriage $(5.4 \%)$. In general, there was no significant differences $(P=0.061)$ in knowledge between pharmaceutical personnel (pharmacists and pharmaceutical technicians) and non-pharmaceutical personnel (nurse assistants and sales persons) regarding contraindications of ALu during the first trimester of pregnancy. Drug dispensers who refused to dispense ALu in the first trimester of 
pregnancy advised for alternative antimalarial drugs including quinine (20.7\%), sulfadoxinepyrimethamine $(35.6 \%)$, dihydroartemisinin-piperaquine $(19.5 \%)$, amodiaquine $(8 \%)$ and sulphalene-pyrimethamine $(9 \%)$ (Table 1$)$.

More than half $(55.5 \%)$ of the pharmacists knew that quinine is the recommended antimalarial drug to be used during the first trimester of pregnancy. Most of the other drug dispensers indicated that sulfadoxine-pyrimethamine, amodiaquine, dihydroartemisininpiperaquine and sulphalene-pyrimethamine were the alternative drugs to be used during the first trimester of pregnancy.

Table 1: The percentage of drug dispensers as to the suggested alternative antimalarial drugs for use during the first trimester of pregnancy $(\mathrm{N}=76)$

\begin{tabular}{|c|c|c|c|c|c|}
\hline $\begin{array}{l}\text { Alternative } \\
\text { antimalarials }\end{array}$ & Pharmacists & $\begin{array}{l}\text { Pharmaceutical } \\
\text { technicians }\end{array}$ & $\begin{array}{l}\text { Nurse } \\
\text { assistants }\end{array}$ & Sales persons & $P$-value \\
\hline Quinine & $55.5 \%$ & $16.6 \%$ & $22.2 \%$ & $5.5 \%$ & 0.3242 \\
\hline $\begin{array}{l}\text { Sulfadoxine- } \\
\text { pyrimethamine }\end{array}$ & $19.4 \%$ & $22.6 \%$ & $32.5 \%$ & $25.9 \%$ & 0.7130 \\
\hline $\begin{array}{l}\text { Dihydroartemisinin- } \\
\text { piperaquine }\end{array}$ & $17.6 \%$ & $23.5 \%$ & $23.5 \%$ & $35.3 \%$ & 0.2342 \\
\hline Amodiaquine & - & $25.0 \%$ & - & $75.0 \%$ & 0.1100 \\
\hline $\begin{array}{l}\text { Sulphalene- } \\
\text { pyrimethamine }\end{array}$ & - & $33.3 \%$ & $16.6 \%$ & $50.0 \%$ & 0.1100 \\
\hline
\end{tabular}

Only 29 (14.5\%) of the drug dispensers had high knowledge, 76 (38.0\%) had medium knowledge and 95 (47.5\%) had low knowledge regarding use of ALu during pregnancy (Table 2). When dispensers were asked if ALu could be used in the second and third trimesters of pregnancy, $124(62 \%)$ said that it is indicated, $72(36 \%)$ said it cannot be used and $4(2 \%)$ were not sure. Forty four $(22 \%)$ drug dispensers mentioned the importance of fatty meals when a patient is taking ALu, indicating that fatty meals enhance absorption of the medicine.

Table 2: Knowledge levels of drug dispensers on the use of ALu during pregnancy $(n=200)$

\begin{tabular}{lllllllll}
\hline \multirow{2}{*}{$\begin{array}{l}\text { Level of } \\
\text { knowledge }\end{array}$} & \multicolumn{2}{l}{ Pharmacists } & \multicolumn{2}{c}{$\begin{array}{c}\text { Pharmaceutical } \\
\text { Technicians }\end{array}$} & \multicolumn{2}{c}{ Nurse assistants } & \multicolumn{2}{c}{ Sales persons } \\
& No. & $\%$ & No. & $\%$ & No. & $\%$ & No. & $\%$ \\
\hline High & 12 & $41.4 \%$ & 4 & $13.8 \%$ & 10 & $34.5 \%$ & 3 & $10.3 \%$ \\
Medium & 32 & $42.1 \%$ & 11 & $14.5 \%$ & 29 & $38.2 \%$ & 4 & $5.3 \%$ \\
Low & 16 & $16.8 \%$ & 19 & $20.0 \%$ & 32 & $33.7 \%$ & 28 & $29.5 \%$
\end{tabular}

\section{Pregnant women}

A total of 200 pregnant women seeking for malaria treatment were interviewed. Majority $(48.5 \%)$ of pregnant women were in the age group of $19-25$ years and $(62 \%)$ of them had attained primary level of education (Table 3). 
Table 3: Socio-demographic characteristics of pregnant women $(N=200)$

\begin{tabular}{llll}
\hline Variable & Response & No. pregnant women & Percent \\
\hline Age group (years) & $\leq 18$ & 13 & 6.5 \\
& $19-25$ & 97 & 48.5 \\
& $26-30$ & 48 & 24.0 \\
& $31-35$ & 30 & 15.0 \\
Level of education & $\geq 36$ & 12 & 6.0 \\
& Primary & 124 & 62.0 \\
& Secondary & 36 & 18.0 \\
Current trimester & No formal education & 40 & 20.0 \\
& First & 23 & 11.5 \\
& Second & 96 & 48.0 \\
Parity & Third & 81 & 40.5 \\
& First pregnancy & 68 & 34.0 \\
& Second pregnancy & 86 & 43.0 \\
& Third or more pregnancy & 46 & 23.0 \\
\hline
\end{tabular}

The results show that age $(P=0.5261)$, trimester of pregnancy $(P=0.1390)$ and parity $(P=0.3642)$ did not have significant influence on the knowledge of pregnant women regarding the use of ALu during pregnancy. Pregnant women who had experienced malaria during their pregnancy mentioned that they used ALu (31.3\%), quinine (23.1\%), sulfadoxine-pyrimethamine (26.9\%), sulphalene-pyrimethamine (15.6\%) and amodiaquine (3.1\%) for treatment of malaria (Figure 1).

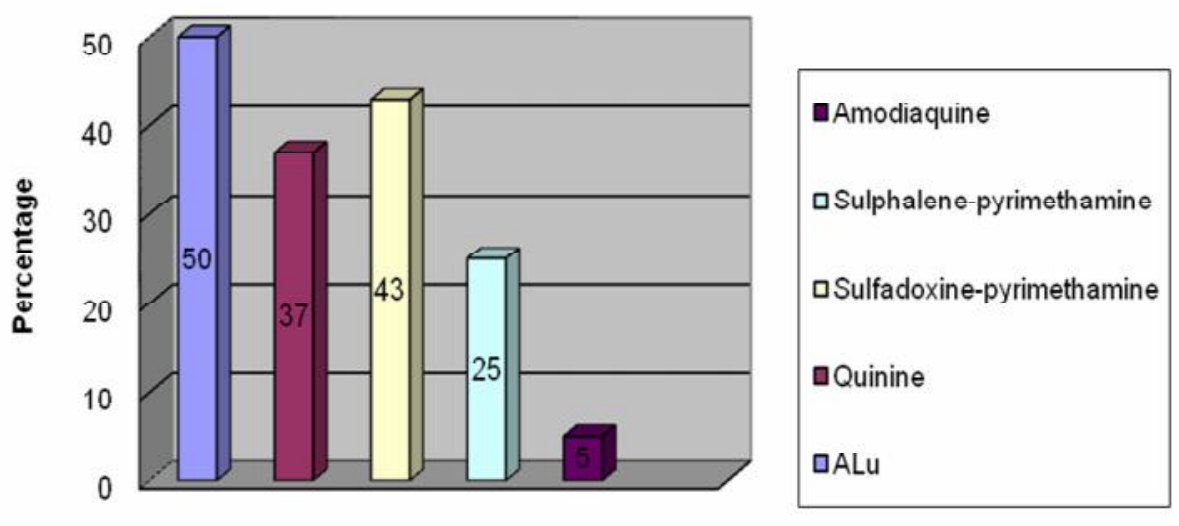

Antimalarials previouslyused by pregnant women

Figure 1: Antimalarial drugs used previously by pregnant women $(\mathrm{n}=160)$

Assessment of knowledge among pregnant women for the use of ALu during pregnancy shows that $19(9.5 \%)$ had high knowledge, $85(42.5 \%)$ had medium knowledge and $96(48.0 \%)$ had low knowledge. The level of education of the pregnant women had correlation with their knowledge about use of ALu during pregnancy $(P=0.0002)$. 
Among pregnant women who had taken antimalarial drugs before pregnancy, 67 (41.9\%) said that they did not experience any side effect with ALu and 93 (58.1\%) said they experienced dizziness (43.6\%), vomiting (32.7\%), hypotension (25.5\%) and fatigue (32.7\%). Seventy-two (36.0\%) of the pregnant women perceived ALu as a good drug for management of malaria. Ninety two (46\%) indicated that they will accept to use it if no other alternative drugs were available. On the other hand, $36(18.0 \%)$ pregnant women perceived ALu as unsafe during pregnancy, and that it has complicated dosage regimen which makes adherence to medication a problem. Twenty two $(11.0 \%)$ pregnant women mentioned that they did not understand the instructions given by the dispensers regarding ALu dosage and duration of use. Majority $(89.0 \%)$ of them said that they understood the instructions. However, 75 (42.2\%) of those who said that they understood the instructions given by dispensers gave incorrect information during interview on the dosage and duration of ALu for treatment of malaria.

Only $9(4.5 \%)$ pregnant women said that they were given information on fatty meals to be taken when taking ALu. However, majority of pregnant women (95.5\%) indicated that they were not given any information on fatty meals and were only told to increase intake of water and fruits when taking ALu. One hundred and sixty three $(81.5 \%)$ pregnant women said that they were asked about the age of their pregnancy before they were given ALu by the dispensers. Thirty three $(16.5 \%)$ of the pregnant women were aware that ALu is not indicated during the first trimester of pregnancy, while 167(83.5\%) were not aware. Among the pregnant women who were aware that ALu should not be taken during the first trimester of pregnancy, 8 (21.9\%) knew that quinine is the alternative medicine. The rest $25(78.1 \%)$ did not know which drug is recommended for the treatment of malaria in the first trimester of pregnancy.

\section{Discussion}

In many countries, especially in the developed world, community pharmacies have been used successfully for the purpose of obtaining over-the-counter drugs and prescription medicines (Toovey, 2006). If properly utilized, pharmacies and drug stores could be the most reliable entry points for educating patients on drug use, especially in malaria-risk groups such as pregnant women. Thus, if well trained dispensing personnel working in community pharmacies can play a key role in providing correct information about antimalarial drug use during pregnancy. However, drug dispensers who are responsible in counselling patients on drug use and other drug information need to be knowledgeable on how to advise patients on rational use of medicines.

Unless the benefits outweigh the risks, it is recommended that artemisinin should not be used in the first trimester of pregnancy because of its teratogenic effects including foetal resorption (Clark et al., 2004). In this study, about a third of the drug dispensers were willing to dispense ALu during the first three months of pregnancy. Since ALu is a newly introduced antimalarial drug in Tanzania, these results imply that drug dispensers have not been trained on the use of ALu during pregnancy. About half of the dispensing personnel working in the community retail pharmacies were non-pharmaceutical personnel. As a result, in some cases patients are not provided with correct information about dosage, side effects and adherence to medications. 
In Dar es Salaam, almost all private retail community pharmacies dispense ALu to patients including pregnant women. The number of patients buying ALu from these facilities is increasing because of the general perception among the public that ALu is of much good quality than the generic ALu which is dispensed in the public health facilities. In addition, many patients in Dar es Salaam prefer to buy antimalarial drugs from private community pharmacies because of easy accessibility and less waiting time. There is therefore a need for training dispensing personnel about the correct use of ALu by the general public and in special groups of people such as pregnant women and children.

Lack of knowledge for antimalarial drug use during pregnancy is a serious problem, especially in areas of intense transmission where antimalarial drugs are given repeatedly to treat frequent fevers (even in the absence of malaria), thus increasing the risk of resistance and adverse drug reactions (Guerin et al., 2002). More often, antimalarial drugs are purchased from pharmacies in the absence of confirmed diagnosis, using drugs obtained without a prescription (Talusina et al., 2002). In this study, only one-fifth of drug dispensers who refused to dispense ALu during the first trimester of pregnancy knew that quinine was the recommended drug. The rest thought that sulfadoxine-pyrimethamine, dihydroartemisinin-piperaquine, amodiaquine and sulphalene-pyrimethamine were the drugs to be used. In addition, $38 \%$ of the dispensers did not know that ALu is a drug of choice for treatment of malaria in the second and third trimester of pregnancy. About a quarter of drug dispensers did not know the correct dosage and duration for ALu. This is an indication that there is irrational dispensing and use of antimalarials, which may lead to dispensing the wrong drugs, under- or over-dosing of patients, thus leading to drug resistance or toxic effects.

About one fifth of the pregnant women in this study perceived ALu as unsafe drug for use during pregnancy. This is likely due to lack of proper counselling of the patients by the care providers about the benefits of ALu in the treatment of malaria. Unlike sulfadoxinepyrimethamine which was taken as a single dose, ALu is a multi-dose regimen taken over three days. For many patients, this makes adherence a problem. There is also a need for routine pharmacovigilance of ALu in order to collect data on possible ALu-related adverse effects (Lang et al., 2006). Case reports and case series are the first source of information to detect adverse events in pregnancy, specific pharmaco-epidemiological studies using a cohort or case-control approach are required to evaluate teratogenic risks.

Food, especially dietary fat enhances the bioavailability of lumefantrine. This is particularly relevant in view of the low food intake by many patients during the acute phase of malaria. It is known that in patients with uncomplicated malaria, the extent and variability of lumefantrine absorption improved with clinical recovery as normal food intake is resumed (Ezzet et al., 1998). In the present study, less than a quarter of drug dispensers in the community pharmacies advised pregnant women about the importance of fatty meals when using ALu. This is an indication that majority of drug dispensers have low knowledge on the benefits of taking ALu with fatty meals in the diet to enhance absorption of lumefantrine.

Although the majority of pregnant women indicated that they understood the instructions, some of them gave incorrect information during interview on the dosage and duration of ALu for treatment of malaria. The short dispensing time, patient work load and lack of continuing education among dispensers might have contributed to patients not getting 
enough and correct information on drug use from the dispensers. In conclusion, the results show that most drug dispensers have inadequate knowledge about good dispensing practice of ALu in pregnancy. There is therefore a need for continuing training of drug dispensers regarding antimalarial drugs use in pregnancy.

\section{Acknowledgements}

We acknowledge the invaluable assistance provided by the medical in-charge of the Dar es Salaam municipal hospitals for this study. We thank Tanzania Food and Drugs Authority for providing a list of retail private community pharmacies in Dar es Salaam. Community pharmacy owners are acknowledged for granting permission to conduct the study in their premises. We also acknowledge the willingness and time spent by pregnant women in providing responses to study questions.

\section{References}

Clark, R.L., White, T.E., A Clode, S., Gaunt, I., Winstanley, P. \& Ward, S.A. (2004) Developmental toxicity of artesunate and an artesunate combination in the rat and rabbit. Birth Defects Research Part B: Developmental and Reproductive Toxicology 71, 380-394.

Draper, C.C., Brubaker, G., Gesser, A., Kilimali, V.A. \& Wernsdorfer, W.H. (1985) Serial studies on the evolution of chloroquine resistance in an area of East Africa receiving intermittent malaria chemosuppression. Bulletin of the World Health Organization 63, 108-118.

Draper, C.C., Hills, M., Kilimali, V.A. \& Brubaker, G. (1988) Serial studies on the evolution of drug resistance in malaria in an area of East Africa: Findings from 1979 up to 1986. Journal of Tropical Medicine and Hygiene 91, 265-273.

Ezzet, F., Mull, R. \& Karbwang, J. (1998) Population pharmacokinetics and therapeutic response of CGP 56697 (artemether + benflumetol) in malaria patients. British Journal of Clinical Pharmacology 46, 553-561.

Guerin, P.J., Olliaro, P., Nosten, F., Druilhe, P., Laxminarayan, R., Binka, F., Kilama, W.L., Ford, N., \& White, N.J. (2002) Malaria: current status of control, diagnosis, treatment, and a proposed agenda for research and development. Lancet Infectious Diseases 2, 564-573.

Hetzel, M.W., Obrist, B., Lengeler, C., Msechu, J.J., Nathan, R., Dillip, A., Makemba, A., Mshana C., Schulze, A., \& Mshinda, H (2008) Obstacles to prompt and effective malaria treatment lead to low community-coverage in two rural districts of Tanzania. BMC Public Health 8, 317.

Kilimali, V.A.E.B. \& Mkufya, A.R. (1985) In vivo and in vitro assessment of the sensitivity of Plasmodium falciparum to chloroquine in four districts of Tanga Region, Tanzania. Transactions of the Royal Society of Tropical Medicine and Hygiene 79, 478-481.

Kitua, A.Y. (1999) Antimalarial drug policy in Tanzania: making a systematic change. Lancet $354,2$.

Kokwaro, G., Mwai, L., \& Nzila, A. (2007) Artemether-lumefantrine in the treatment of uncomplicated falciparum malaria. Expert Opinion on Pharmacotherapy 8, 75-94. 
Laffan, S., James, A., Maleeff, B., Pagana, J., Bushdid, P., Clark, R. \& White, T. (2006) Mitochondrial involvement of artesunate toxicity in rat embryonic erythroblasts. Abstract: $46^{\text {th }}$ annual meeting of the Teratology Society; Tucson, Arizona.

Lang, T., Hughes, D., Kanyok, T., Kengeya-Kayondo, J., Marsh, V., Haaland, A., Pirmohamed, M., \& Winstanley, P. (2006) Beyond registration - measuring the public-health potential of new treatments for malaria in Africa. Lancet Infectious Diseases 6, 46-52.

McGready, R., Stepniewska, K., Lindegardh, N., Ashley, E.A., La, Y., Singhasivanon. P., White, N.J., \& Noste, F. (2006) The pharmacokinetics of artemether and lumefantrine in pregnant women with uncomplicated falciparum malaria. European Journal Clinical Pharmacology 62, 1021-1031.

Minzi, O.M., Haule, A.F. (2008) Poor knowledge on new malaria treatment guidelines among drug dispensers in private pharmacies in Tanzania: the need for involving the private sector in policy preparations and implementation. East African Journal of Public Health 5: 117-121.

MoHSW (2006) National Guidelines for Malaria Diagnosis and Treatment. Malaria Control Series 11. Ministry of Health and Social Welfare, Dar es Salaam, Tanzania.

Mugittu, K., Ndejembi, M., Malisa, A., Lemnge, M., Premji, Z., Mwita, A., Nkya, W., Kataraihya, J., Abdulla, S., Beck, H.P. \& Mshinda, H. (2004) Therapeutic efficacy of sulfadoxinepyrimethamine and prevalence of resistance markers in Tanzania prior to revision of malaria treatment policy: Plasmodium falciparum dihydrofolate reductase and dihydropteroate synthase mutations in monitoring in vivo resistance.

Rønn, A., Msangeni, H. \& Mhina, J. (1996) High level of resistance of Plasmodium falciparum to salfadoxine-pyrimethamine in children in Tanzania. Transactions of the Royal Society of Tropical Medicine and Hygiene 54, 197-181.

Talusina, A.O., Langi, P., Bakyaita, N., Egwang, T., Mutabingwa, T.K., Watkins, W., van Marck, E., \& D'Alessandro, U. (2002) Intensity of malaria transmission, antimalarial drug use and resistance in Uganda: what is the relationship between these three factors? Transactions of the Royal Society of Tropical Medicine and Hygiene 96, 310-317.

Toovey, S. (2006) Malaria chemoprophylaxis advice: survey of South African community pharmacists' knowledge and practices. Journal of Travel Medicine 13, 161-165.

van Vugt, M., Looareesuwan, S., Wilairatana, P., McGready, R., Villegas, L., Gathmann, I., Mull, R., Brockman, A., White, N.J., \& Nosten, F. (2000) Artemether-lumefantrine for the treatment of multidrug-resistant falciparum malaria. Transactions of the Royal Society of Tropical Medicine and Hygiene 94, 545-548.

Weiss, M.C., Booth, A., Jones, B., Ramjeet, S. \& Wong, E. (2010) Use of simulated patients to assess the clinical and communication skills of community pharmacists. Pharmaceutical World Science 32: 353-361.

White, N.J., van Vugt, M., \& Ezzet, F. (1999) Clinical pharmacokinetics and pharmacodynamics of artemether-lumefantrine. Clinical Pharmacokinetics, 37, 105-125.

WHO/AFRO (2004) A strategic framework for malaria prevention and control during pregnancy in the African region. 
Worrall, E., Morel, C., Yeung, S., Borghi, J., Webster, J., Hill, J., Wiseman, V., \& Mills, A. (2007) The economics of malaria in pregnancy - a review of the evidence and research priorities. Lancet Infectious Diseases 7, 156-168. 